Authors' accepted manuscript: IEEE International Symposium on Robot and Human Interactive Communication (RO-MAN), 2017.

\title{
Integrating Olfaction in a Robotic Telepresence Loop*
}

\author{
Javier Monroy, Francisco Melendez-Fernandez, Andres Gongora and Javier Gonzalez-Jimenez
}

\begin{abstract}
In this work we propose enhancing a typical robotic telepresence architecture by considering olfactory and wind flow information in addition to the common audio and video channels. The objective is to expand the range of applications where robotics telepresence can be applied, including those related to the detection of volatile chemical substances (e.g. land-mine detection, explosive deactivation, operations in noxious environments, etc.). Concretely, we analyze how the sense of smell can be integrated in the telepresence loop, covering the digitization of the gases and wind flow present in the remote environment, the transmission through the communication network, and their display at the user location. Experiments under different environmental conditions are presented to validate the proposed telepresence system when localizing a gas emission leak at the remote environment.
\end{abstract}

\section{INTRODUCTION}

Robotic telepresence is an emerging technology with a great potential in applications such as elder assistance, surveillance or inspection of dangerous areas. A robotic telepresence system results from the combination of information and communication technologies (ICTs) and mobile robotic solutions. On the one hand, ICTs permit users to remotely control mobile robots within realistic environments, overcoming the still limited performance of autonomous robots in these scenarios [1]. On the other hand, mobile robots help in enhancing the capabilities of traditional ICTs, enabling users to interact within the robot environment as if they were physically present [2].

Typically, robotic telepresence relies on the human senses of vision, hearing and, when haptic devices are available, the sense of touch. This standard configuration has allowed telepresence systems to be successfully deployed in applications such as health care [3], [4], elder assistance [5], or visual inspection of disaster areas [6], [7]. Moreover, the prevalence of high quality video conferencing using mobile devices, tablets and portable computers has enabled a drastic growth in telepresence robots to enhance the virtual experience of being physically present at the office [8], [9].

Yet, despite this enhanced "virtual" presence, applications exist that require detecting and measuring other entities or variables of the remote environment. This is the case of olfaction-related applications, where information about the volatile chemical substances and the wind flow in the environment must be provided. Example applications include

\footnotetext{
*This work has been funded by Governments of Spain and Andalusia, and the European Regional Development Fund under project TEP530.

Authors are with the MAchine Perception and Intelligent Robotics group (MAPIR), and the Instituto de Investigacion Biomedica de Malaga (IBIMA). University of Malaga, Spain. Corresponding author: jgmonroyeuma.es
}

land-mine detection, operations in dangerous areas due to the presence of hazardous chemicals, or explosive deactivation, among others. Furthermore, traditional telepresence applications that rely only on videoconferencing, can indeed enhance the user feeling of being at the remote location by complementing these senses with olfaction [10], [11].

Telepresence demands the users' senses to be provided with such stimuli as to give the feeling of immersion in the remote site. This requires to capture, transmit, and finally display each stimuli in a proper way: images/video for visual awareness, sound for auditory perception, or forces to simulate the feeling of touch. This is not a simple task, and many researchers worldwide seek improving how to digitize, transmit and deliver such information to the tele-operator in order to enhance its virtual experience.

In the case of olfaction, electronic noses (e-noses) are the devices aimed at mimicking the humans' sense of smell by means of an array of gas sensors and machine learning algorithms [12], [13]. In a nutshell, e-noses provide an artificial representation of the airborne volatiles present in the environment. Naturally, the spectrum of volatiles and the concentrations that an e-nose can detect strongly depends on the sensors it hosts, so a specific design and configuration are usually necessary to adapt to the application requirements. In this regard, novel e-nose designs as the presented in [14] aim at providing a modular architecture to simplify this customization process.

E-noses have been successfully employed in conjunction with autonomous mobile robots to carry out tasks like: gas source localization [15], [16], gas distribution mapping [17], [18] or chemical recognition [19]-[21]. In many cases, olfaction is complemented with wind flow perception by means of $2 \mathrm{D}$ or $3 \mathrm{D}$ anemometers, since the dispersion of chemical volatiles is strongly correlated with the later. Nevertheless, given the high complexity of real environments, autonomous systems are not yet fully operational, and human intervention is required in most practical scenarios. Therefore, the integration of olfactory information in a telepresence system seems a logical and intuitive line of actuation, allowing a human operator to take over the reasoning on the remotely sensed data.

Regarding to how the digitized data is presented to the user, there are many different screens, virtual-reality goggles, surround sound systems, haptic devices, etc, but there are just a few olfactory and wind-flow delivering devices. This makes explicit one of the main problems of introducing olfaction in the telepresence loop, the great difficulty to convey the sensed stimuli to the user. When compared to vision, where 
Authors' accepted manuscript: IEEE International Symposium on Robot and Human Interactive Communication

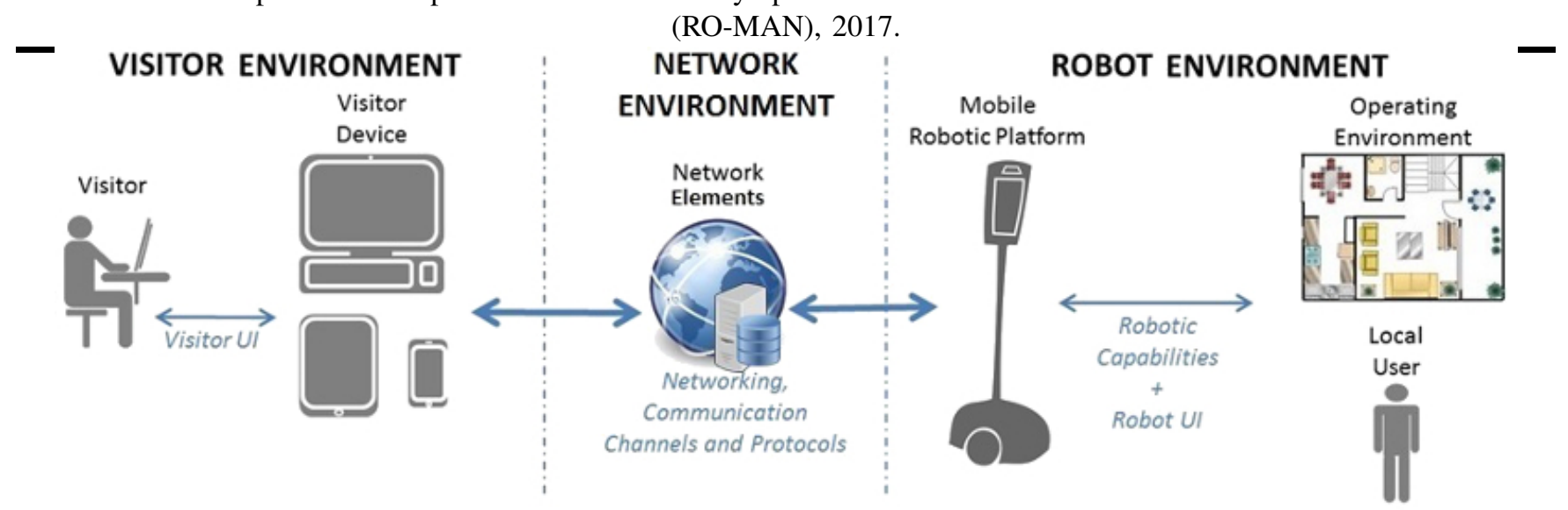

Fig. 1: Robotic telepresence systems entails three environments: visitor, network, and robot environments.

all the information can be precisely represented with the well-known RGB color components, we face the problem that there not exist such a simple odor space, but it is believed that the human sense of smell has approximately 400 dimensions [22]. Moreover, to reproduce a scent from its digitized representation, the olfactory display would need hundred of different primary-odors and some mechanism to properly combine them in order to generate a similar stimuli [23].

According to how the odorous stimuli is presented to the user, we can differentiate two types of olfactory displays: ubiquitous type, which deliver the smell to the environment, and wearable type, which are worn by the user [11]. The former provides users with the ability to sense scents without wearing encumbering and obtrusive devices on the face or head, but makes more challenging to switch the scent within a short time because of the difficult to dissipate the previous scent [24]. In this regard, some researchers have proposed the use of sophisticate "air cannons" [25] to deliver a clump of smell to a location near the user's nose, reducing the volume of scented air released and therefore the time span at which two different scents can be delivered. On the other hand, wearable type displays accurately present the odor directly to or near the user's nose, enabling its use in large spaces, including outdoor environments [26]. Their main limitations reside in the reduced set of smells that can be generated and the short operation time, in order to keep the system wearable. Yet, there are some initiatives that propose analyzing the user's breathing pattern to reduce the amount of scent delivered, enabling the use of the display for a longer period [27].

In this work we analyze how the sense of olfaction can be introduced in the telepresence loop to enhance the user experience and to increase the range of applications where this technology can be employed. We cover the digitization of the volatiles and wind flow present at the remote environment, the transmission between the robot and the tele-operator, and the display of this new information. We propose a basic, yet illustrative implementation of an olfactory telepresence system, and present experiments to analyze its validity.

\section{Overview of the Robotic Telepresence System}

Independently to the application domain, robotic telepresence systems, in general, comprise three environments (see Fig. 1): the robot environment, where a mobile robotic platform endowed with different sensor modalities, enables the virtual visit to the robot workplace, the visitor environment, where a networked device provides the visitor with an interface to interact with the robot, and a network environment that supports the communication between visitors and robots.

In this work we employ the robotic telepresence architecture presented in [28], because of its modularity and ease of implementation. This generic architecture endorses the telepresence application with the following features:

- It provides an open-source, web-based user interface for robot teleoperation, which enriches the solution with interesting features like cross-platform compatibility, accessibility and maintainability. The interface relies on NodeJS, HTML5 and WebRTC technologies to enable these features.

- It integrates efficient and robust middleware communications based on the IoT protocols, HTTP, WebSockets, and MQTT. The use of these standards provides wellstructured mechanisms for communication as well as it permits the inclusion of third-party components or plugins.

- It includes interfaces with robotic control architectures, based on, but not limited to, the widely used ROS [29] and MOOS [30] robotic frameworks.

The three components of this architecture are detailed next:

\section{A. Network Infrastructure}

The network infrastructure relies on a client/server architecture to support the communications between visitors an robots, and if necessary, to provide security mechanisms and data persistence. The server is implemented in NodeJS, a runtime environment designed to develop javascript serverside applications, which offers a rich set of readily available 
Authors' accepted manuscript: IEEE International Symposium on Robot and Human Interactive Communication (RO-MAN), 2017.

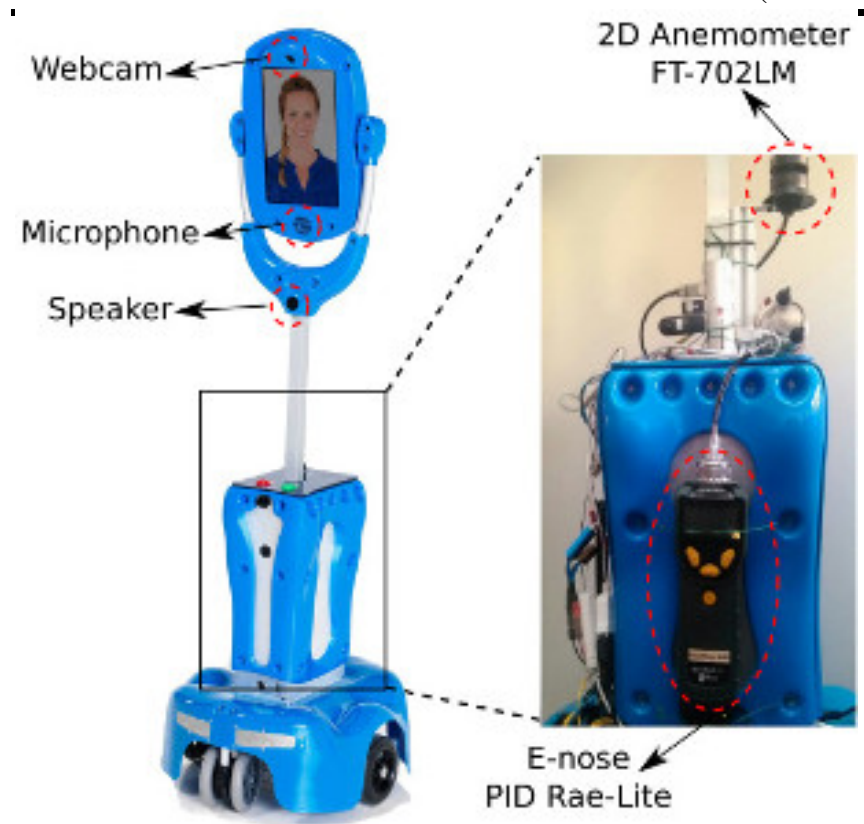

Fig. 2: Picture of the Giraff mobile robot and description of the on-board sensors for the olfactory robotic telepresence.

utilities through a package ecosystem. It offers two local services, namely, a message broker enabling the server application to communicate with clients using the publish/subscribe messaging pattern [31], and a database to store application data and session logs. The utilities selected to implement these services have been Mosquitto MQTT Message Broker ${ }^{1}$ and MongoDB Database ${ }^{2}$. The server transmits and receives $\mathrm{A} / \mathrm{V}$ data over websockets using the $\mathrm{WebRTC}^{3} \mathrm{API}$, and relies on the MQTT data channel to exchange robotic control data.

\section{B. Robotic Platform}

In this work we consider the commercial telepresence robot Giraff (see Fig. 2), which is built upon a manufacturerdeveloped base, endowed with a videoconference set and a tilting head. The hardware of the standard Giraff has been enhanced with a laser scanner, an e-nose for gas detection, and a 2D ultrasonic anemometer for wind flow measurement (see Section III for a detailed description). It is controlled through a ROS-based robotic architecture, including a controller for the robotic base, MQTT compatible communications, and self-localization capabilities.

\section{Visitor Display}

The employed robotic telepresence architecture enables the use of diverse visitor devices, i.e laptop, desktop, tablet, including different peripherals to capture the user events, i.e., keyboard, mouse, and touch screens, and media displays with varied size and different resolutions. The user interface

\footnotetext{
${ }^{1}$ http://mqtt.org/

${ }^{2}$ https://www.mongodb.com/

${ }^{3}$ https://webrtc.org/
}

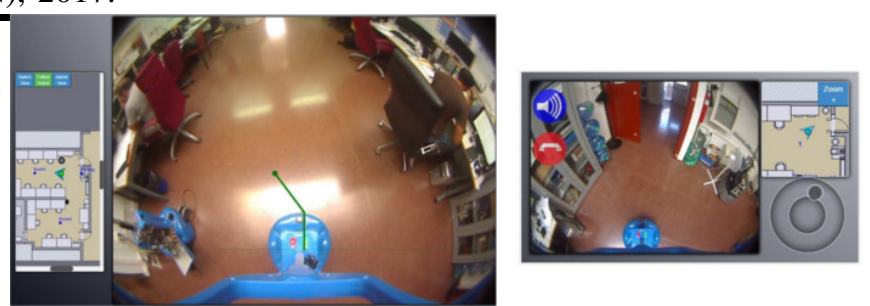

Fig. 3: Visitor UI layout. Left) layout for medium and large media devices. Right) layout for small media devices.

is provided through a WebRTC compatible web browser, providing the following features:

- Cross-platform compatibility without installing additional plugins, nor software. The web-based visitor interface deals efficiently with hardware, visualization and communication issues.

- Instant access and updates. Since the user interface is delivered online by the central server, visitors can retrieve the most recent version by just accessing the server URL.

- Simple and responsive design suitable for multiple sizes and resolutions in the visitor device. The interface provides different views, automatically adapted to the client device, and integrates controllers for keyboard, mouse and touchscreen events.

Figure 3 displays a view of the standard visitor interface (i.e without olfaction). Presentation elements comprise a minimalist layout composed of two viewports. The main one renders the video stream received from the robot environment and overlays some elements, e.g., graphic helpers, application alerts and/or status messages. The secondary viewport displays a schematic map of the robot environment with the aim of improving the visitor situation awareness. The map includes a floorplan of the robot environment enriched with some structural elements, along with the robot pose.

\section{The Olfaction Components in the TElepresence ARChiteCture}

Building on the generic telepresence architecture presented in Section II, we now integrate olfaction and wind flow data in the loop. Concretely, we analyze the sensors, the transmission requirements, and the displays involved to present these two new information sources to the user. For an overview of the proposed robotic telepresence architecture, see Fig. 4.

\section{A. Olfaction}

As previously introduced, e-nose is the technology which aims at mimicking the humans' sense of smell. E-noses are gas-sensing devices composed by an array of non-selective gas sensors whose joint response comprise the so called "fingerprint" of the odor (i.e a distinctive multidimensional response pattern). This odor-fingerprint is not human-legible, nor has a direct correlation with the composition of the gas mixture (i.e each gas sensor in the array responds to multiple gases, so it is not possible to get the gas composition from the raw sensors response). This entails that, in most 
Authors' accepted manuscript: IEEE International Symposium on Robot and Human Interactive Communication

VISITOR ENVIRONMENT (RO-MAN), 2017.

NETWORK

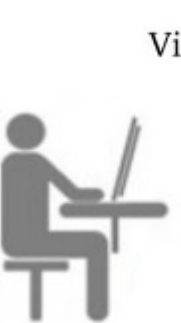
NETWORK
ENVIRONMENT

Visual display

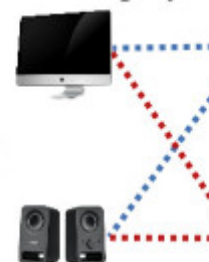

Sound display

MQTT Data

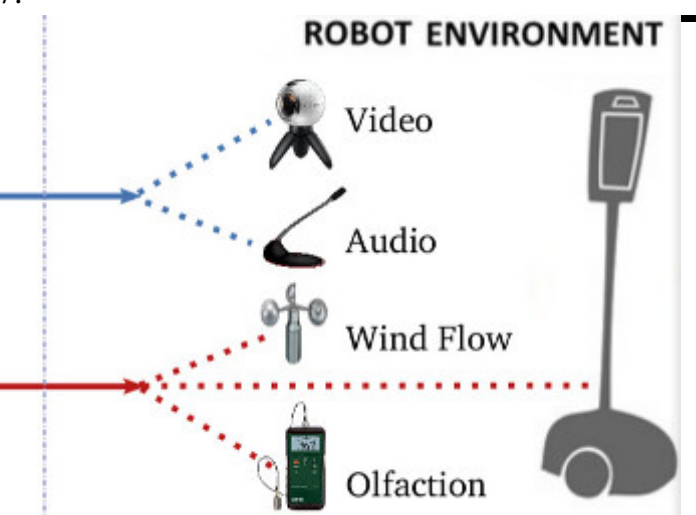

Fig. 4: Our Robotic telepresence system with olfaction and wind flow information. Both information sources are transmitted through the MQTT data channel and presented to the user over the standard visual and sound displays.

cases, the odor-fingerprint must be processed by machine learning algorithms to infer the volatiles identities and their concentration. Whether to process the data at the remote or local environment depends on the particular characteristics of the application at hand: robots' computational capacity, bandwidth of the communication network, preferred user display (mobile, tablet, PC), etc.

Finally, the digitized olfaction data must be displayed to the user in a proper way so to contribute with the virtual presence feeling. However, and despite the recent advances in this line, there is no yet an olfactory display able to provide the olfactory stimuli the user would perceive if it were physically present at the robot environment. Moreover, we seek to maximize the range of client devices that can be used for teleoperation (PCs, tablets, smartphones...), providing an interface not relying upon specific hardware. Therefore, we propose here a minimalist olfaction display which accounts just for visual and sound stimuli under the assumption that only one scent is of interest for the telepresence activity. More specifically, we rely on the always present visual display to show a bar-plot proportional to the inferred gas concentration (see Fig. 5), and simultaneously generate an audible sound signal with a cadence proportional to the gas concentration (i.e similar to a Geiger counter for radiation measurement). This second display allows the user to perceive the gas concentration without having to lose focus on the robot navigation. For scenarios where more than one scent is of interest to the user, the e-nose data must be previously classified to determine the components of the scent and their concentration. In this case, auditory stimuli is inadvisable, and the visual display shows the name of the most probable odor-class (and its \% probability) in conjunction with the concentration.

\section{B. Wind Flow}

For detecting and presenting to the user the wind flows present at the robot environment, we employ an anemometer which provides the wind speed and direction. This is a very lightweight information so there is basically no need to preprocess the data.
As in the case of olfactory information, the display is the bottleneck of the system. Though some initiatives have been proposed to force airflows at the user environment by means of fans [32], they are still on very immature state. We therefore choose to display the wind information visually. The simplest option would be to include in the user interface a wind compass where the wind vector is plotted. This approach present the drawback of having different reference systems for the wind flow (following the standard N-S-E$\mathrm{W}$ schema) and the robot (which continuously changes as it moves). The latter leads in most cases to confusion more than an improvement of the telepresence feeling. Consequently, for the experiments carried out in this work, we transform the wind vector to the robot's reference system, and plot a vector over the robot location indicating the wind direction and strength that the robot is exposed to.

\section{EXPERIMENTS}

This section presents an experiment for the validation of the proposed robotic telepresence system consisting on the localization of a gas leak in a remote environment. The objective is to determine if the proposed architecture is suitable for the execution of olfaction-related tasks in telepresence applications. To that end, more than 60 testruns have been recorded where a gas leak in an office-like environment has to be localized.

One important limitation when validating olfaction-related proposals is the high variability of environmental conditions in real scenarios, and their significant influence over the dispersal of gases [33]. This entails that repeatability among runs of the same experiment usually yield very different results, making difficult to validate the system. To overcame this issue, gas dispersal simulation based on computational fluid dynamics (CFD) tools is usually employed [34], [35], allowing an accurately control of the gas dispersion, and consequently enabling comparison among repetitions. Following this approach, we generated a simulated version of an office-like environment (composed of four rooms and multiple furniture), and employed OpenFoam CFD ${ }^{4}$

\footnotetext{
${ }^{4}$ http://www.openfoam.com/
} 
Authors' accepted manuscript: IEEE International Symposium on Robot and Human Interactive Communication (RO-MAN), 2017.

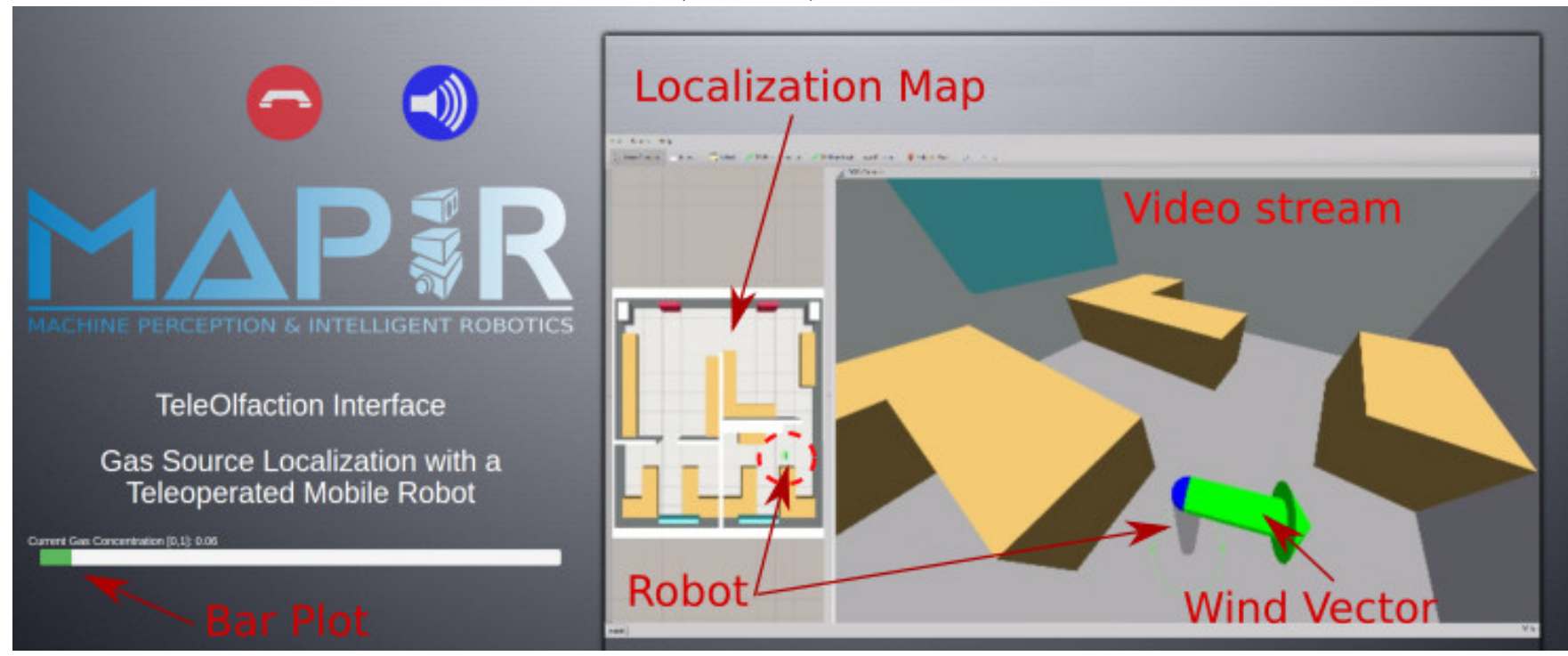

Fig. 5: Proposed visual interface for the robotic telepresence system. In addition to the common video stream and localization map, gas concentration is displayed by means of a colored bar-plot together with an audible signal. Accordingly, the wind strength and direction is displayed by a wind vector at the current robot location.

to generate four different scenarios, varying the wind flow conditions and gas source location (see Fig. 6). The simulated CFD data was then integrated with a standard $\operatorname{ROS}^{5}$ robotic simulator, to provide gas and wind measurements in the virtual environment (for further details on the simulator and the experimental setup, please see [36] and [35]). It must be stressed that only the robot environment was simulated, retaining the same visitor and network environments of the proposed telepresence loop. That is, the user interacted with the system as if he/she was teleoperating a real robot (see Fig. 5).

Table I summarizes the success ratio (i.e. when the user pinpoint the gas source position at less than $1 \mathrm{~m}$ from the real location) and average time needed to complete the localization task with the proposed telepresence architecture. As can be appreciated, we tested two sensor configurations: olfaction alone, and olfaction plus wind flow, with the aim to to analyze the influence of the latter in the localization process. From these results we can infer that the overall success ratio worsens when wind information is provided to the user (possibly due to the lack of training and understanding of how the wind flow affects the gas dispersal in a 3D environment), but the average search time improves, with a notable reduction of $25 \%$. In either case, these results demonstrate that the presented architecture does indeed enable the development of olfaction-related applications through telepresence. Finally, the low success rate obtained in these experiments suggests that there is plenty room for improvement, mostly on the olfactory interface.

\section{CONCLUSions}

In this work we have proposed a telepresence architecture which accounts not only for the common visual and hearing

${ }^{5}$ http://www.ros.org

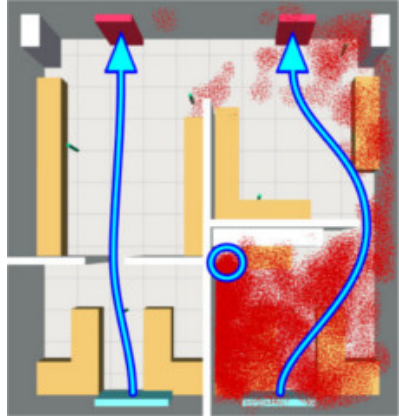

(a) Scenario 1

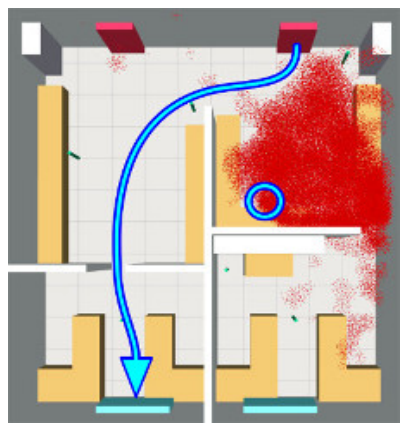

(c) Scenario 3

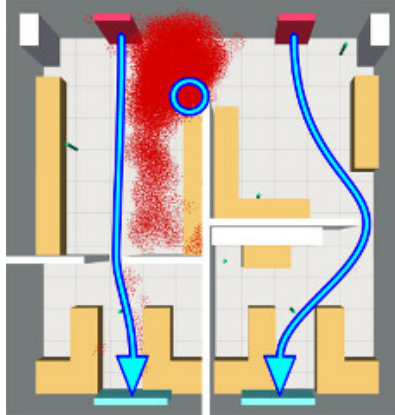

(b) Scenario 2

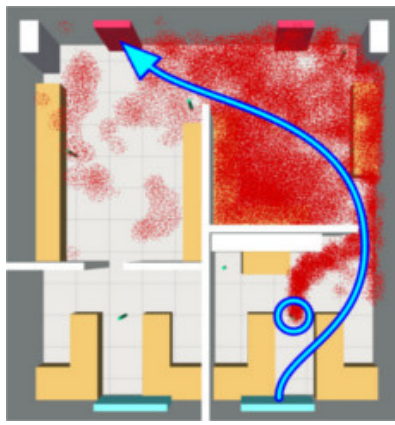

(d) Scenario 4

Fig. 6: The experimental scenario and its different environmental configurations. Each figure shows a 3D representation of the environment, the main wind flow (blue arrows), the gas source location (blue circle), and the simulated gas distribution after a time period of 100 s (red point-cloud).

feedback, but also olfaction and wind flow information at the remote environment. We have analyzed the challenges and open issues when related to the digitization and display of these magnitudes, and proposed a minimalist user interface 
Authors' accepted manuscript: IEEE International Symposium on Robot and Human Interactive Communication (RO-MAN), 2017.

TABLE 1: Overall success ratio and average time to locate the emission source (at $1.5 \mathrm{~m}$ threshold) in the simulated environment.

\begin{tabular}{|r|c|c|} 
& \multicolumn{1}{c}{ Success ratio } & Average time \\
\hline olfaction & $21 / 34$ & $211.2 \mathrm{~s}$ \\
\cline { 2 - 3 } olfaction + wind & $16 / 31$ & $180.6 \mathrm{~s}$ \\
\hline
\end{tabular}

which relys on the always present video and sound stimuli. Experimental validation with more than 60 test-runs demonstrate that robotic telepresence is a suitable technology to solve olfaction-related applications.

For future research we plan to improve the olfaction display to provide a more inmmersive experience, and to analyze how the user behaviour may be affected by how this information is presented. The long-term goal is to learn the human reasoning related to olfaction (e.g. when localizing a gas leak), and apply it to autonomous robots.

\section{REFERENCES}

[1] J. Y. Chen and M. J. Barnes, "Supervisory control of multiple robots effects of imperfect automation and individual differences," Human Factors: The Journal of the Human Factors and Ergonomics Society, vol. 54, no. 2, pp. 157-174, 2012.

[2] S. O. Adalgeirsson and C. Breazeal, "Mebot: a robotic platform for socially embodied presence," in Proceedings of the 5th ACM/IEEE international conference on Human-robot interaction. IEEE Press, 2010, pp. 15-22.

[3] R. Leeb, L. Tonin, M. Rohm, L. Desideri, T. Carlson, and J. d. R. Millán, "Towards independence: a BCI telepresence robot for people with severe motor disabilities," Proceedings of the IEEE, vol. 103, no. 6, pp. 969-982, 2015.

[4] A. Kristoffersson, S. Coradeschi, and A. Loutfi, "A review of mobile robotic telepresence," Advances in Human-Computer Interaction, vol. 2013, p. 3, 2013.

[5] S. Coradeschi, A. Cesta, G. Cortellessa, L. Coraci, C. Galindo, J. Gonzalez, L. Karlsson, A. Forsberg, S. Frennert, F. Furfari, A. Loutfi, A.and Orlandini, F. Palumbo, F. P., S. von Rump, A. Stimec, J. Ullberg, and B. Otslund, "Giraffplus: a system for monitoring activities and physiological parameters and promoting social interaction for elderly," in Human-Computer Systems Interaction: Backgrounds and Applications 3. Springer International Publishing, 2014, pp. 261-271.

[6] A. Halme, J. Suomela, and M. Savela, "Applying telepresence and augmented reality to teleoperate field robots," Robotics and autonomous systems, vol. 26, no. 2-3, pp. 117-125, 1999.

[7] R. R. Murphy, S. Tadokoro, and A. Kleiner, "Disaster robotics," in Springer Handbook of Robotics. Springer, 2016, pp. 1577-1604.

[8] W.-C. Wen, H. Towles, L. Nyland, G. Welch, and H. Fuchs, "Toward a compelling sensation of telepresence: Demonstrating a portal to a distant (static) office," in Visualization 2000. Proceedings. IEEE, 2000, pp. 327-333.

[9] K. M. Tsui, M. Desai, H. A. Yanco, and C. Uhlik, "Exploring use cases for telepresence robots," in International Conference on Human-Robot Interaction (HRI). ACM/IEEE, 2011, pp. 11-18.

[10] T. Moon and G. J. Kim, "Design and evaluation of a wind display for virtual reality," in Proceedings of the ACM symposium on Virtual reality software and technology. ACM, 2004, pp. 122-128.

[11] Y. Chen, "Olfactory display: development and application in virtual reality therapy," in Artificial Reality and Telexistence-Workshops, 2006. ICAT'06. 16th International Conference on. IEEE, 2006, pp. $580-584$.

[12] R. Dutta, E. L. Hines, J. W. Gardner, and P. Boilot, "Bacteria classification using cyranose 320 electronic nose," Biomedical engineering online, vol. 1, no. 1, p. 4, 2002.

[13] J. Gonzalez-Jimenez, J. Monroy, and J.-L. Blanco, "The multi-chamber electronic nose - an improved olfaction sensor for mobile robotics," Sensors, vol. 11, no. 6, pp. 6145-6164, 2011.
[14] C. Sanchez-Garrido, J. Monroy, and J. Gonzalez-Jimenez, "A configurable smart e-nose for spatio-temporal olfactory analysis," in IEEE Sensors, oct 2014, pp. 1968-1971.

[15] W. Jatmiko, K. Sekiyama, and T. Fukuda, "A pso-based mobile robot for odor source localization in dynamic advection-diffusion with obstacles environment: theory, simulation and measurement," IEEE Computational Intelligence Magazine, vol. 2, no. 2, pp. 37-51, 2007.

[16] S. Soldan, G. Bonow, and A. Kroll, "Robogasinspector - a mobile robotic system for remote leak sensing and localization in large industrial environments: Overview and first results," IFAC Proceedings Volumes, vol. 45, no. 8, pp. 33-38, 2012, 1st IFAC Workshop on Automatic Control in Offshore Oil and Gas Production.

[17] J. Monroy, J.-L. Blanco, and J. Gonzalez-Jimenez, "Time-variant gas distribution mapping with obstacle information," Autonomous Robots, vol. 40, no. 1, pp. 1-16, 2016.

[18] J.-L. Blanco, J. Monroy, J. Gonzalez-Jimenez, and A. Lilienthal, "A Kalman filter based approach to probabilistic gas distribution mapping," in Proceedings of the 28th Annual ACM Symposium on Applied Computing, 2013, pp. 217-222.

[19] J. Monroy and J. Gonzalez-Jimenez, "Gas classification in motion: An experimental analysis," Sensors \& Actuators: B. Chemical, vol. 240, pp. $1205-1215,2017$.

[20] F. W. Grasso and J. Atema, "Integration of flow and chemical sensing for guidance of autonomous marine robots in turbulent flows,' Environmental Fluid Mechanics, vol. 2, no. 1-2, pp. 95-114, 2002.

[21] F.-M. Schleif, B. Hammer, J. Monroy, J. Gonzalez-Jimenez, J.-L. Blanco, M. Biehl, and N. Petkov, "Odor recognition in robotics applications by discriminative time-series modeling," Pattern Analysis and Applications, vol. 19, no. 1, pp. 207-220, 2016.

[22] B. Malnic, P. A. Godfrey, and L. B. Buck, "The human olfactory receptor gene family," Proceedings of the National Academy of Sciences of the United States of America, vol. 101, no. 8, pp. 2584-2589, 2004.

[23] M. Meister, "On the dimensionality of odor space," Elife, vol. 4, p. e07865, 2015.

[24] H. Matsukura, T. Yoneda, and H. Ishida, "Smelling screen: development and evaluation of an olfactory display system for presenting a virtual odor source," IEEE transactions on visualization and computer graphics, vol. 19, no. 4, pp. 606-615, 2013.

[25] Y. Yanagida, S. Kawato, H. Noma, A. Tomono, and N. Tesutani, "Projection based olfactory display with nose tracking," in Virtual Reality, 2004. Proceedings. IEEE. IEEE, 2004, pp. 43-50.

[26] T. Nakamoto and H. P. D. Minh, "Improvement of olfactory display using solenoid valves," in Virtual Reality Conference, 2007. VR'07. IEEE. IEEE, 2007, pp. 179-186.

[27] T. Yamada, S. Yokoyama, T. Tanikawa, K. Hirota, and M. Hirose, "Wearable olfactory display: Using odor in outdoor environment," in Virtual Reality Conference, 2006. IEEE, 2006, pp. 199-206.

[28] F. Melendez-Fernandez, C. Galindo, and J. Gonzalez-Jimenez, "A web-based solution for robotic telepresence," International Journal of Advanced Robotic Systems, Under Review.

[29] M. Quigley, K. Conley, B. Gerkey, J. Faust, T. Foote, J. Leibs, R. Wheeler, and A. Y. Ng, "Ros: an open-source robot operating system," in ICRA workshop on open source software, vol. 3, no. 3.2, 2009 , p. 5.

[30] P. M. Newman, "Moos-mission orientated operating suite," Massachusetts Institute of Technology, Tech. Rep, vol. 2299, no. 08, 2008.

[31] P. T. Eugster, P. A. Felber, R. Guerraoui, and A.-M. Kermarrec, "The many faces of publish/subscribe," ACM Computing Surveys (CSUR), vol. 35, no. 2, pp. 114-131, 2003.

[32] H. Matsukura, T. Nihei, and H. Ishida, "Multi-sensorial field display: Presenting spatial distribution of airflow and odor," in Virtual Reality Conference (VR), 2011 IEEE. IEEE, 2011, pp. 119-122.

[33] B. I. Shraiman and E. D. Siggia, "Scalar turbulence," Nature, vol. 405 , no. 6787 , pp. 639-646, 2000.

[34] Z. Liu and T.-F. Lu, "A simulation framework for plume-tracing research," in Australasian Conference on Robotics and Automation, 2008

[35] J. Monroy, V. Hernandez-Bennets, H. Fan, A. Lilienthal, and J. Gonzalez-Jimenez, "GADEN: A 3D gas dispersion simulator for mobile robot olfaction in realistic environments," MDPI Sensors, 2017, Under review.

[36] A. Gongora, J. Monroy, and J. Gonzalez-Jimenez, "A robotic experiment toward understanding human gas-source localization strategies," in International Symposium on Olfaction and Electronic Nose (ISOEN), 2017 